九州大学学術情報リポジトリ

Kyushu University Institutional Repository

\title{
Quasimodular solutions of a differential equation of hypergeometric type
}

Kaneko, Masanobu

Graduate School of Mathematics, Kyushu University

Koike, Masao

Graduate School of Mathematics, Kyushu University

http://hdl. hand le. net/2324/20460

出版情報: Developments in mathematics. 11, pp.329-336, 2003. Kluwer Academic Publishers バージョン：

権利関係 : 


\title{
Quasimodular solutions of a differential equation of hypergeometric type
}

\author{
Masanobu Kaneko and Masao Koike
}

\section{$\S 1$. Introduction And Main TheOrem}

In our previous paper [2], we studied further the solutions of the following differential equation in the upper half-plane $\mathfrak{H}$ which was originally found and studied in [4] in connection with the arithmetic of supersingular elliptic curves;

$$
f^{\prime \prime}(\tau)-\frac{k+1}{6} E_{2}(\tau) f^{\prime}(\tau)+\frac{k(k+1)}{12} E_{2}^{\prime}(\tau) f(\tau)=0
$$

Here, $k$ is an integer or half an integer, the symbol ' denotes the differentiation $(2 \pi i)^{-1} d / d \tau=q \cdot d / d q\left(q=e^{2 \pi i \tau}\right)$, and $E_{2}(\tau)$ is the "quasimodular" Eisenstein series of weight 2 for the full modular group $\mathrm{SL}_{2}(\mathbb{Z})$ :

$$
E_{2}(\tau)=1-24 \sum_{n=1}^{\infty}\left(\sum_{d \mid n} d\right) q^{n}
$$

Let $p \geq 5$ be a prime number and $F_{p-1}(\tau)$ be the solution of the above differential equation for $k=p-1$ which is modular on $\mathrm{SL}_{2}(\mathbb{Z}$ ) (such a solution exists and is unique up to a scalar multiple). For any zero $\tau_{0}$ in $\mathfrak{H}$ of the form $F_{p-1}(\tau)$, the value of the $j$ function at $\tau_{0}$ is algebraic and its reduction modulo (an extension of) $p$ is a supersingular $j$-invariant of characteristic $p$, and conversely, all the supersingular $j$-invariants are obtained in this way from the single solution $F_{p-1}(\tau)$ with suitable choices of $\tau_{0}$. This is the arithmetic connection that motivated our study of the differential equation.

Various modular forms on $\mathrm{SL}_{2}(\mathbb{Z})$ and its subgroups were obtained in [2] as solutions to this differential equation, the groups depending on the choice of $k$. Every modular solution is expressed in terms of a hypergeometric polynomial in a suitable modular function (hence the "hypergeometric type" in the title of the paper), also depending on the choice of $k$. For instance, if $k \equiv 0,4 \bmod 12$, we have a modular solution

$$
E_{4}(\tau)^{\frac{k}{4}} F\left(-\frac{k}{12},-\frac{k-4}{12},-\frac{k-5}{6} ; \frac{1728}{j(\tau)}\right)
$$


where

$$
F(a, b, c ; x)=\sum_{n=0}^{\infty} \frac{(a)_{n}(b)_{n}}{(c)_{n}} \frac{x^{n}}{n !}, \quad(a)_{n}=a(a+1) \cdots(a+n-1)
$$

is the Gauss hypergeometric series (which becomes a polynomial when $a$ or $b$ is a negative integer, which is the case here), $E_{4}(\tau)$ the Eisenstein series of weight 4 on $\mathrm{SL}_{2}(\mathbb{Z})$, and $j(\tau)$ the elliptic modular invariant.

In addition to the modular solutions, quite remarkable was an occurrence of a quasimodular form, not of weight $k$ as in the modular case but of weight $k+1$. In the present paper, we give another supply of examples of quasimodular forms as solutions to an analogous differential equation attached to the group $\Gamma_{0}^{*}(2)$, which is not contained in $\mathrm{SL}_{2}(\mathbb{Z})$

$$
\Gamma_{0}^{*}(2)=\left\langle\Gamma_{0}(2),\left(\begin{array}{cc}
0 & -1 \\
2 & 0
\end{array}\right)\right\rangle
$$

where

$$
\Gamma_{0}(2)=\left\{\left(\begin{array}{ll}
a & b \\
c & d
\end{array}\right) \in \mathrm{SL}_{2}(\mathbb{Z}) \mid c \equiv 0 \bmod 2\right\} .
$$

( $\Gamma_{0}^{*}(2)$ is the triangular group " $2 A$ " in the notation of Conway-Norton [1].)

Let

$$
E_{2 A}(\tau):=\left(E_{2}(\tau)+2 E_{2}(2 \tau)\right) / 3=1-8 q-40 q^{2}-32 q^{3}-\cdots
$$

be the quasimodular form of weight 2 on $\Gamma_{0}^{*}(2)$ which is the logarithmic derivative of the form

$$
\Delta_{2 A}(\tau):=\eta(\tau)^{8} \eta(2 \tau)^{8}=q-8 q^{2}+12 q^{3}+64 q^{4}-\cdots
$$

of weight 8 on $\Gamma_{0}^{*}(2) ; E_{2 A}(\tau)=\Delta_{2 A}^{\prime}(\tau) / \Delta_{2 A}(\tau)$, an analogous situation in the $S L_{2}(\mathbb{Z})$ case where $E_{2}(\tau)$ is the logarithmic derivative of the Ramanujan $\Delta(\tau)$. Consider the following differential equation;

$$
(\#)_{k} \quad f^{\prime \prime}(\tau)-\frac{k+1}{4} E_{2 A}(\tau) f^{\prime}(\tau)+\frac{k(k+1)}{8} E_{2 A}^{\prime}(\tau) f(\tau)=0 .
$$

Solutions which are modular on the group $\Gamma_{0}^{*}(2)$ and its subgroups were studied in $[6,7]$. In particular, when $k$ is a non-negative integer congruent to 0 or 6 modulo 8 , the equation $(\#)_{k}$ has a one dimensional space of solutions which are modular on the group $\Gamma_{0}^{*}(2)$ itself. We note here that the equation $(\#)_{k}$ has a characterization by the invariance of the space of solutions under the action of $\Gamma_{0}^{*}(2)$, similar to the previous case for $\mathrm{SL}_{2}(\mathbb{Z})$, owing to the fact that there is no holomorphic modular form of weight 2 on $\Gamma_{0}^{*}(2)$ (see $[5]$ and $[2, \S 5]$ ). By a general theory of ordinary differential equations, we see that the equation $(\#)_{k}$ has a quasimodular solution (which, since its transformation under $\tau \rightarrow-1 / 2 \tau$ is also a solution, inevitably gives a solution having $\log q$ term in the expansion at $q=0$ ) only when $k$ is a positive integer congruent to 3 modulo 4 . 
In the following, we show there indeed exists a quasimodular solution in this case and describe explicitly the solution in terms of a certain orthogonal polynomials. First we need to develop some notations. Put

$$
\begin{aligned}
C(\tau) & :=2 E_{2}(2 \tau)-E_{2}(\tau) \\
& =1+24 \sum_{n=1}^{\infty}\left(\sum_{\substack{d \mid n \\
d: \text { odd }}} d\right) q^{n}=1+24 q+24 q^{2}+96 q^{3}+\cdots \\
D(\tau) & :=\frac{\eta(2 \tau)^{16}}{\eta(\tau)^{8}}=\sum_{n=1}^{\infty}\left(\sum_{\substack{d \mid n \\
d: \text { odd }}}(n / d)^{3}\right) q^{n}=q+8 q^{2}+28 q^{3}+64 q^{4}+\cdots
\end{aligned}
$$

where

$$
\eta(\tau)=q^{\frac{1}{24}} \prod_{n=1}^{\infty}\left(1-q^{n}\right)=q^{\frac{1}{24}}-q^{\frac{25}{24}}-q^{\frac{49}{24}}+q^{\frac{121}{24}}+\cdots
$$

is the Dedekind eta function. The functions $C(\tau)$ and $D(\tau)$ are modular forms of respective weights 2 and 4 on the group $\Gamma_{0}(2)(=" 2 B$ ") and the graded ring of modular forms of integral weights on $\Gamma_{0}(2)$ is generated by these $C(\tau)$ and $D(\tau)$. Recall that (see [3]) an element of degree $k$ in the graded ring $\mathbb{C}\left[E_{2}(\tau), C(\tau), D(\tau)\right]$, where the generators $E_{2}(\tau), C(\tau), D(\tau)$ have degrees 2, 2, and 4 respectively, is referred to as a quasimodular form of weight $k$ (on $\Gamma_{0}(2)$ ). Incidentally, the graded ring of modular forms of integral weights on $\Gamma_{0}^{*}(2)$ is generated by three elements $C(\tau)^{2}=\left(E_{4}(\tau)+\right.$ $\left.4 E_{4}(2 \tau)\right) / 5, C(\tau)^{3}-128 C(\tau) D(\tau)=\left(E_{6}(\tau)+8 E_{6}(2 \tau)\right) / 9$, and $\Delta_{2 A}(\tau)$ of respective weights $4,6,8$, of which $C(\tau)^{2}$ and $\Delta_{2 A}(\tau)$ generate freely the subring consisting forms of weight being multiple of 4 , and the whole space as a graded module is generated over this ring by $C(\tau)^{3}-128 C(\tau) D(\tau)$.

Now define a sequence of polynomials $P_{n}(x)(n=0,1,2, \ldots)$ by

$$
P_{0}(x)=1, P_{1}(x)=x, \quad P_{n+1}(x)=x P_{n}(x)+\lambda_{n} P_{n-1}(x) \quad(n=1,2, \ldots)
$$

where

$$
\lambda_{n}=4 \frac{(4 n+1)(4 n+3)}{n(n+1)}
$$

First few examples are

$$
P_{2}(x)=x^{2}+70, P_{3}(x)=x^{3}+136 x, P_{4}(x)=x^{4}+201 x^{2}+4550, \ldots
$$

The $P_{n}(x)$ is even or odd polynomial according as $n$ is even or odd. We also define a second series of polynomials $Q_{n}(x)$ by the same recursion (with different initial values):

$$
Q_{0}(x)=0, Q_{1}(x)=1, \quad Q_{n+1}(x)=x Q_{n}(x)+\lambda_{n} Q_{n-1}(x) \quad(n=1,2, \ldots),
$$

a couple of examples being

$$
Q_{2}(x)=x, Q_{3}(x)=x^{2}+66, Q_{4}(x)=x^{3}+131 x, \ldots
$$

The $Q_{n}(x)$ has opposite parity: It is even if $n$ is odd and odd if $n$ is even.

Put $G(\tau)=C(\tau)^{2}-128 D(\tau)\left(=\left(4 E_{4}(2 \tau)-E_{4}(\tau)\right) / 3\right)$. 
Theorem. Let $k=4 n+3(n=0,1,2, \ldots)$. The following quasimodular form of weight $k+1$ on $\Gamma_{0}(2)$ is a solution of $(\#)_{k}$ :

$$
{\sqrt{\Delta_{2 A}(\tau)}}^{n} P_{n}\left(\frac{G(\tau)}{\sqrt{\Delta_{2 A}(\tau)}}\right) \frac{C^{\prime}(\tau)}{24}-{\sqrt{\Delta_{2 A}(\tau)}}^{n+1} Q_{n}\left(\frac{G(\tau)}{\sqrt{\Delta_{2 A}(\tau)}}\right) .
$$

Remark. The appearance of the square root $\sqrt{\Delta_{2 A}(\tau)}$ in the formula is superficial because of the parities of $P_{n}(x)$ and $Q_{n}(x)$, that is, the form is actually an element in $\mathbb{Q}\left[E_{2}(\tau), C(\tau), D(\tau)\right]$, by noting $\Delta_{2 A}(\tau)=D(\tau)\left(C(\tau)^{2}-64 D(\tau)\right)$ and $C^{\prime}(\tau)=\left(E_{2}(\tau) C(\tau)-C(\tau)^{2}\right) / 6+32 D(\tau)$. The form does not belong to $\Gamma_{0}^{*}(2)$.

\section{§2. Proof of Theorem}

It is convenient to introduce the operator $\vartheta_{k}$ defined by

$$
\vartheta_{k}(f)(\tau)=f^{\prime}(\tau)-\frac{k}{8} E_{2 A}(\tau) f(\tau)
$$

By the quasimodular property of $E_{2}(\tau)$ or the fact that $E_{2 A}(\tau)$ is the logarithmic derivative of $\Delta_{2 A}(\tau)$, we have the transformation formulas

$$
E_{2 A}\left(\frac{a \tau+b}{c \tau+d}\right)=(c \tau+d)^{2} E_{2 A}(\tau)+\frac{4}{\pi i} c(c \tau+d) \quad\left(\left(\begin{array}{ll}
a & b \\
c & d
\end{array}\right) \in \Gamma_{0}(2)\right)
$$

and

$$
E_{2 A}\left(-\frac{1}{2 \tau}\right)=2 \tau^{2} E_{2 A}(\tau)+\frac{8}{\pi i} \tau
$$

From these we see that if $f$ is modular of weight $k$ on a subgroup of $\Gamma_{0}^{*}(2)$, then $\vartheta_{k}(f)$ is modular of weight $k+2$ on the same group. If $f$ and $g$ have weights $k$ and $l$, the Leibniz rule

$$
\vartheta_{k+l}(f g)=\vartheta_{k}(f) g+f \vartheta_{l}(g)
$$

holds. We sometimes drop the suffix of the operator $\vartheta_{k}$ when the weights of modular forms we consider are clear. With this operator, the equation $(\#)_{k}$ can be rewritten as

$$
\left(\#^{\prime}\right)_{k} \quad \vartheta_{k+2} \vartheta_{k}(f)(\tau)=\frac{k(k+2)}{64} C(\tau)^{2} f(\tau)
$$

(use $\left.E_{2 A}^{\prime}(\tau)=\left(E_{2 A}(\tau)^{2}-C(\tau)^{2}\right) / 8\right)$.

Denote the form in the theorem by $F_{k}(\tau)$. We first establish the recurrence relation $($ note $n=(k-3) / 4)$ :

$$
F_{k+4}(\tau)=G(\tau) F_{k}(\tau)+\lambda_{n} \Delta_{2 A}(\tau) F_{k-4}(\tau)
$$


This is a consequence of the recursion of $P_{n}$ and $Q_{n}$, namely (we often omit the variable $\tau$ hereafter)

$$
\begin{aligned}
& G F_{k}+\lambda_{n} \Delta_{2 A} F_{k-4} \\
& =G\left({\sqrt{\Delta_{2 A}}}^{n} P_{n}\left(\frac{G}{\sqrt{\Delta_{2 A}}}\right) \frac{C^{\prime}}{24}-{\sqrt{q \Delta_{2 A}}}^{n+1} Q_{n}\left(\frac{G}{\sqrt{\Delta_{2 A}}}\right)\right) \\
& +\lambda_{n} \Delta_{2 A}\left({\sqrt{\Delta_{2 A}}}^{n-1} P_{n-1}\left(\frac{G}{\sqrt{\Delta_{2 A}}}\right) \frac{C^{\prime}}{24}-{\sqrt{\Delta_{2 A}}}^{n} Q_{n-1}\left(\frac{G}{\sqrt{\Delta_{2 A}}}\right)\right) \\
& ={\sqrt{\Delta_{2 A}}}^{n+1}\left(\frac{G}{\sqrt{\Delta_{2 A}}} P_{n}\left(\frac{G}{\sqrt{\Delta_{2 A}}}\right)+\lambda_{n} P_{n-1}\left(\frac{G}{\sqrt{\Delta_{2 A}}}\right)\right) \frac{C^{\prime}}{24} \\
& -{\sqrt{\Delta_{2 A}}}^{n+2}\left(\frac{G}{\sqrt{\Delta_{2 A}}} Q_{n}\left(\frac{G}{\sqrt{\Delta_{2 A}}}\right)+\lambda_{n} Q_{n-1}\left(\frac{G}{\sqrt{\Delta_{2 A}}}\right)\right) \\
& ={\sqrt{\Delta_{2 A}}}^{n+1} P_{n+1}\left(\frac{G}{\sqrt{\Delta_{2 A}}}\right) \frac{C^{\prime}}{24}-{\sqrt{\Delta_{2 A}}}^{n+2} Q_{n+1}\left(\frac{G}{\sqrt{\Delta_{2 A}}}\right) \\
& =F_{k+4} \text {. }
\end{aligned}
$$

Now we prove by induction that the $F_{k}(\tau)$ satisfies the equation $\left(\#^{\prime}\right)_{k}$. We can check the cases $k=3$ and 7 directly. Assume $F_{k-4}$ and $F_{k}$ satisfy $\left(\#^{\prime}\right)_{k-4}$ and $\left(\#^{\prime}\right)_{k}$ respectively. Then by using (1) and the formulas

$$
\vartheta(C)=-\frac{1}{4} G, \quad \vartheta(G)=-\frac{1}{2} C^{3}, \quad \vartheta\left(\Delta_{2 A}\right)=0
$$

we have

$$
\begin{aligned}
\vartheta^{2}\left(F_{k}\right) & =\vartheta\left(\vartheta\left(F_{k}\right) G-\frac{1}{2} C^{3} F_{k}\right)+\lambda_{n} \Delta_{2 A} \vartheta^{2}\left(F_{k-4}\right) \\
& =\vartheta^{2}\left(F_{k}\right) G-\frac{1}{2} \vartheta\left(F_{k}\right) C^{3}+\frac{3}{8} C^{2} G F_{k}-\frac{1}{2} C^{3} \vartheta\left(F_{k}\right)+\lambda_{n} \Delta_{2 A} \vartheta^{2}\left(F_{k-4}\right) \\
& =\frac{k(k+2)}{64} C^{2} G F_{k}-C^{3} \vartheta\left(F_{k}\right)+\frac{3}{8} C^{2} G F_{k}+\frac{(k-4)(k-2)}{64} \lambda_{n} \Delta_{2 A} C^{2} F_{k-4} \\
& =\frac{k^{2}+2 k+24}{64} C^{2} G F_{k}+\frac{(k-4)(k-2)}{64} \lambda_{n} \Delta_{2 A} C^{2} F_{k-4}-C^{3} \vartheta\left(F_{k}\right) .
\end{aligned}
$$

Hence we find

$$
\begin{aligned}
& \vartheta^{2}\left(F_{k+4}\right)-\frac{(k+4)(k+6)}{64} C^{2} F_{k+4} \\
& =\left(\frac{k^{2}+2 k+24}{64}-\frac{(k+4)(k+6)}{64}\right) C^{2} G F_{k} \\
& +\left(\frac{(k-4)(k-2)}{64}-\frac{(k+4)(k+6)}{64}\right) \lambda_{n} \Delta_{2 A} C^{2} F_{k-4} \\
& =-C^{2}\left(\frac{k}{8} G F_{k}+C \vartheta\left(F_{k}\right)+\frac{k+1}{4} \lambda_{n} \Delta_{2 A} F_{k-4}\right) .
\end{aligned}
$$


The proof of the theorem therefore boils down to show the equation

$$
\frac{k}{8} G F_{k}+C \vartheta\left(F_{k}\right)=-\frac{k+1}{4} \lambda_{n} \Delta_{2 A} F_{k-4}
$$

For this we also proceed by induction. For $k=7$ the equation is checked directly. Assuming that this is valid for $k$, we have

$$
F_{k+4}=G F_{k}+\lambda_{n} \Delta_{2 A} F_{k-4}=\frac{1}{2(k+1)}\left((k+2) G F_{k}-8 C \vartheta\left(F_{k}\right)\right)
$$

and

$$
\begin{aligned}
& \frac{k+4}{8} G F_{k+4}+C \vartheta\left(F_{k+4}\right) \\
& =\frac{k+4}{16(k+1)} G\left((k+2) G F_{k}-8 C \vartheta\left(F_{k}\right)\right) \\
& +\frac{1}{2(k+1)} C\left(-\frac{1}{2}(k+2) C^{3} F_{k}+(k+2) G \vartheta\left(F_{k}\right)+2 G \vartheta\left(F_{k}\right)-8 C \vartheta^{2}\left(F_{k}\right)\right) \\
& =\frac{(k+2)(k+4)}{16(k+1)}\left(G^{2}-C^{4}\right) F_{k} \\
& =-\frac{k+5}{4} \lambda_{n+1} \Delta_{2 A} F_{k} .
\end{aligned}
$$

Here we have used the (previous) induction assumption that $F_{k}$ satisfies $\left(\#^{\prime}\right)_{k}$ and the relation $G^{2}-C^{4}=-256 \Delta_{2 A}$. This completes our proof.

\section{REFERENCES}

1. J. Conway and S. Norton, Monstrous Moonshine, Bull. London Math. Soc. 11 (1979), 308-339.

2. M. Kaneko and M. Koike, On modular forms arising from a differential equation of hypergeometric type, to appear in Ramanujan J.

3. M. Kaneko and D. Zagier, A generalized Jacobi theta function and quasimodular forms, "The Moduli Space of Curves", Progress in Math. 129 (1995), Birkhäuser, 165-172.

4. M. Kaneko and D. Zagier, Supersingular j-invariants, Hypergeometric series, and Atkin's orthogonal polynomials, AMS/IP Studies in Advanced Mathematics 7 (1998), 97-126.

5. M. Koike, Modular forms on non-compact arithmetic triangle groups, preprint.

6. M. Koike and T. Saijo, in preparation.

7. T. Saijo, Modular forms and differential equations, Kyushu University Master's thesis (1999.3).

Graduate School of Mathematics, Kyushu University 33, Fukuoka 812-8581, Japan

E-mail address: mkaneko@math.kyushu-u.ac.jp

Graduate School of Mathematics, Kyushu University, Ropponmatu, Fukuoka 810-8560, JAPAN

E-mail address: koike@math.kyushu-u.ac.jp 